\title{
Patient-Specific Simulation of Internal Defibrillation
}

Daniel Mocanu ${ }^{1}$, Joachim Kettenbach ${ }^{2}$, Michael O. Sweeney ${ }^{3}$, Bruce H. KenKnight ${ }^{4}$, Ron Kikinis ${ }^{2}$, and Solomon R. Eisenberg ${ }^{1}$

1 Department of Biomedical Engineering, Boston University,

Boston, MA, U.S.A.

$\{$ mocanu, sre $\} @ b u . e d u$

http://bme.bu.edu/Fieldsandtissues

2 Surgical Planning Laboratory, Brigham and Women's Hospital,

Boston, MA, U.S.A.

\{kettjo,kikinis\}@bwh.harvard.edu

3 Cardiac Pacing and Implantable Device Therapies, Brigham and Women's Hospital,

Boston, MA, U.S.A.

mosweeney@partners . org

4 Department of Therapy Research, Guidant/CPI,

St.Paul, MN, U.S.A.

bruce.kenknight@guidant.com

\begin{abstract}
The objective of this study is to investigate the predictive capacity of computational models of electrical defibrillation by comparing the results of patient-specific simulations to clinically determined defibrillation metrics. Finite volume models of the thoracic conductive anatomy and in situ electrodes were constructed for seven patients who received implantable defibrillators. These models were based on segmented X-ray CT images taken shortly after implant. The models were solved for electric field (current density) distributions corresponding to a defibrillation shock. The defibrillation parameters were calculated from these distributions based on critical mass and inexcitability criteria for successful defibrillation. Preliminary results show good agreement between clinical and simulated thresholds for four of the seven patients modeled to date. The defibrillation parameters for the remaining three patients are underestimated. The correspondence between the predicted and measured defibrillation metrics observed in four of the seven patients is encouraging and provides preliminary support to the potential utility of the modeling approach. This approach may allow for patient specific presurgical planning, as well as provide a convenient computational testbed for evaluating new electrode configurations. Although these results are promising additional subjects are needed to further validate the modeling method.
\end{abstract}

\section{Introduction}

Ventricular fibrillation (VF) is a severe heart condition that can lead to sudden cardiac death (SCD) if not treated promptly. VF often starts with a premature 
excitatory stimulus during the vulnerable period (rising phase of the $\mathrm{T}$ wave) when the cardiac myocytes are in various states of recovery. The only effective clinical intervention to extinguish VF is electrical defibrillation. With the advent of smaller generators, catheter electrodes and active can technologies, implantable cardioverter defibrillators (ICD) have been shown to be very effective in protecting against SCD and have become the treatment of choice for patients with drug-resistant heart arrhythmias.

Determining the energy that the defibrillator must deliver in order to extinguish fibrillation and return the heart to a normal sinus rhythm continues to be primarily an empirical process. ICD implantation requires induction of VF to set the delivered energy and to confirm that the device can defibrillate at an energy $10 \mathrm{~J}$ below the maximum device output to assure an adequate safety margin. Typically, at least two, and often three, VF inductions are performed. Since each VF induction has some element of risk, including the possibility of non-conversion and death, ICD implantation might be improved by providing an estimate of the patient's defibrillation energy requirement prior to implant.

Previous computer modeling studies 1, 2, 2, 3] have shown a good correlation with the overall mean of reported clinical defibrillation metrics. These findings suggest a possible use for computational models in the presurgical planning of ICD implantation. The goal of our current research is to assess the predictive capacity of patient-specific computer models of internal defibrillation by comparing patient-specific simulated and clinica 11 defibrillation metrics (the current threshold $I_{t h}$, the voltage threshold $V_{t h}$ and the energy threshold DFT). To date, solutions for seven patient-specific models have been completed. These models were created from segmented cross-sectional CT images obtained post-implant from Brigham and Women's Hospital ${ }^{1}$. This paper presents the modeling results, and compares the predicted and clinical defibrillation parameters for the recruited patients.

\section{Methods}

\subsection{Clinical DFT Determination}

The defibrillation threshold (DFT) is defined as the smallest amount of energy that can be delivered to extinguish VF and return the heart to normal sinus rhythm. ICD implantation and DFT testing procedures follow a standardized clinical protocol. In all but one of the patients, the Endotak catheter lead system (Guidant/CPI) was implanted. A similar lead system (Medtronic) was used in the remaining patient. ICDs were implanted in the left pectoral region with venous access via the subclavian vein (Fig. 1). In all cases, the catheter electrode surface in the right ventricle (RV) adjacent to the apex was the cathode. The catheter electrode surface in the superior vena cava (SVC), and the surface of the implanted pulse generator (can) were the anodes. Fluoroscopic imaging

${ }^{1}$ The study was carried out in accordance with the guidelines established by the Human Research Committee at the Brigham and Women's Hospital, Boston MA. 
was used to verify the correct lead placement. Clinical DFT testing was performed following a step-down procedure. VF was induced by applying a pulse of alternating current and the defibrillation shock was delivered 10 seconds later. The defibrillation waveform had a biphasic shape (Fig. 2a), with $60 \%$ tilt in the positive phase and $50 \%$ tilt in the negative phase. The pulse width of the first phase (PW1) and the second phase (PW2) were $60 \%$ and $40 \%$ respectively, of the total waveform duration (typically 10-15 msec). Typical trials started at 20 $\mathrm{J}$ and decremented until VF was no longer terminated (an example is shown in Fig. 2b).

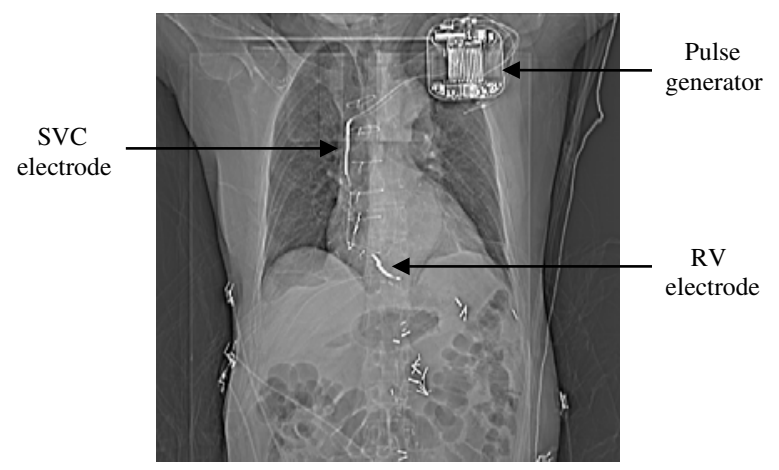

Fig. 1. X-ray image showing the implanted pulse generator and the catheter electrodes in the superior vena cava (SVC) and right ventricle (RV)

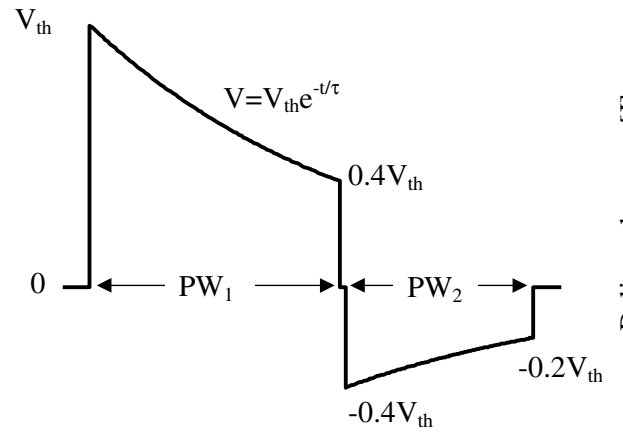

a)

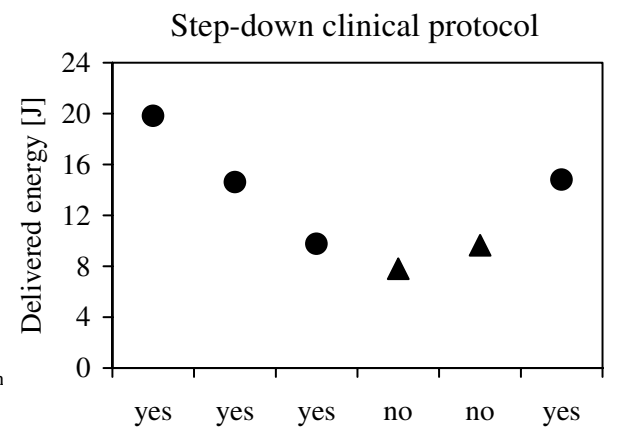

b)

Fig. 2. a) CPI/Guidant biphasic waveform; b) Step-down clinical protocol for DFT testing. Solid circles represent successful shocks. Unsuccessful trials are shown with solid triangles 


\subsection{Model Construction}

All patients were scanned on a spiral CT scanning system (Somatom Plus 4, Siemens Medical Systems, NJ) post-implant with the transvenous catheter electrodes in place. For each patient, nine tissue labels were segmented to generate the following objects: skin, subcutaneous fat, ribs and spine, thoracic wall muscles, lung, mediastinum, heart muscle, aorta and pulmonary vessels, catheter electrodes and ICD can [4]. Each of the 3-D computer models was constructed with a structured meshing algorithm, using low resolution images $(128 \times 128)$ in which each voxel in the segmented image data set was defined as a volume element in the computational model (Fig. 3). The size of a volume element is $3 \times 3 \times 6 \mathrm{~mm}$, with slight variations depending on patient geometrical features. The total number of elements in the models varied between 350,000 and 450,000.

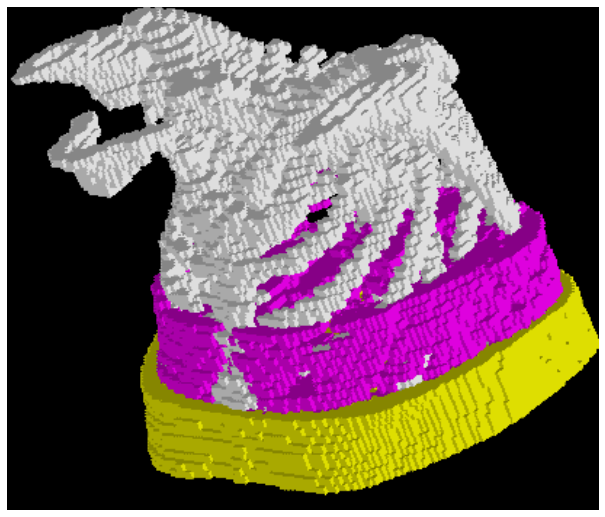

a)

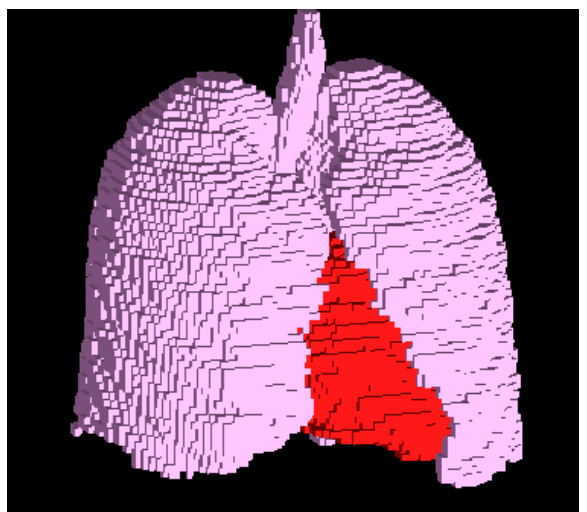

b)

Fig. 3. Voxel based finite volume mesh. For clarity, only indicated parts of the model are shown: a) bone structure and bottom layers of the thoracic wall muscle and fat; b) heart muscle and lungs

\subsection{Computational Approach}

The thoracic anatomy was considered to be a linear, isotropic, piece-wise homogeneous volume conductor having negligible capacitive and inductive properties. Under these assumptions, the electric potential $\phi$ is the solution of the elliptic partial differential equation:

$$
\nabla \cdot(\sigma \nabla \phi)=0
$$

subject to boundary conditions:

$$
\phi=\Phi_{i}
$$


on the electrodes (RV, SVC, can), and

$$
\frac{\partial \phi}{\partial n}=0
$$

on the thorax surface, where $\sigma$ is the electric conductivity, $\Phi_{i}$ is the constant potential on the $i_{t h}$ electrode surface and $n$ is the surface normal. The electrical conductivity values for the six tissues were selected from literature reported estimates [5], 6]: $\sigma_{\text {blood }}=8 \mathrm{mS} / \mathrm{cm}, \sigma_{\text {myocardium }}=2.5 \mathrm{mS} / \mathrm{cm}, \sigma_{\text {muscle }}=2.5$ $\mathrm{mS} / \mathrm{cm}, \sigma_{\text {lung }}=0.7 \mathrm{mS} / \mathrm{cm}, \sigma_{\text {bone }}=0.1 \mathrm{mS} / \mathrm{cm}, \sigma_{\text {fat }}=0.5 \mathrm{mS} / \mathrm{cm}$. Equation (1) was solved using the I-DEAS/TMG software package (Structural Dynamics Research Corporation, Milford, OH, USA) which uses a finite volume approach to find the electric potential distribution. Current density distributions were computed from the potential distribution.

\subsection{Defibrillation Metrics Calculation}

Four defibrillation metrics (impedance $Z$; the current threshold $I_{t h}$, the voltage threshold $V_{t h}$ and the energy threshold DFT) were calculated for each simulation to aid in the interpretation and evaluation of the solutions. The critical mass hypothesis of defibrillation states that in order to extinguish fibrillation wavefronts, a critical mass of the ventricular myocardium has to be exposed to electric fields equal to or greater than the inexcitability threshold $\left(E_{t h}\right)$ required to render a fibrillating myocyte inexcitable. Thus, in this study, each simulation was assumed to defibrillate with the minimum delivered energy that exposed $95 \%$ of the ventricular myocardium to electric fields greater than or equal to $E_{t h}=3.5 \mathrm{~V} / \mathrm{cm} \mathrm{[7],} \mathrm{8].}$

In the simulation, a unit voltage was applied between the RV cathode, and the SVC and can anodes. The resulting electric field magnitudes in the heart muscle were then scaled such that $95 \%$ of the ventricular myocardium was exposed to an electric field equal to or greater than $E_{t h}=3.5 \mathrm{~V} / \mathrm{cm}$. Thus, the voltage threshold $V_{t h}$ and the current threshold $I_{t h}$ are scaled versions of the voltage applied in the simulation and the resulting delivered current. The DFT was calculated from the threshold voltage $V_{t h}$ and threshold current $I_{t h}$ based on the Guidant/CPI biphasic waveform features (Fig. 2a) using equation (4):

$$
D F T=\int_{0}^{1.61 \tau} V_{t h} I_{t h} e^{-2 t / \tau} d t=0.48 C V_{t h}^{2} .
$$

where $\tau=Z C$ is the time constant and $C=150 \mu \mathrm{F}$ is the capacitance of the pulse generator.

\section{Results}

Simulated electric current pathways during a defibrillation shock are shown in Fig. 4. Patient-specific clinical and model-predicted defibrillation metrics (DFT, $I_{t h}, V_{t h}$ and $Z$ ) are compared in Figs. 5a-d. Individual patients are encoded 


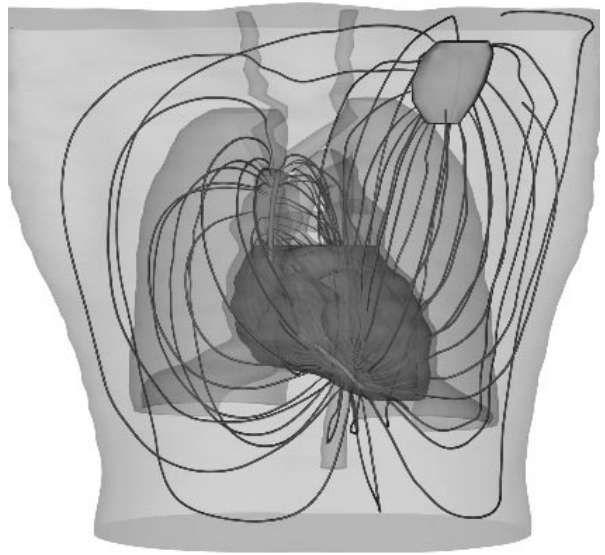

Fig. 4. Flux lines representing the electric current pathways during a defibrillation shock (simulation)

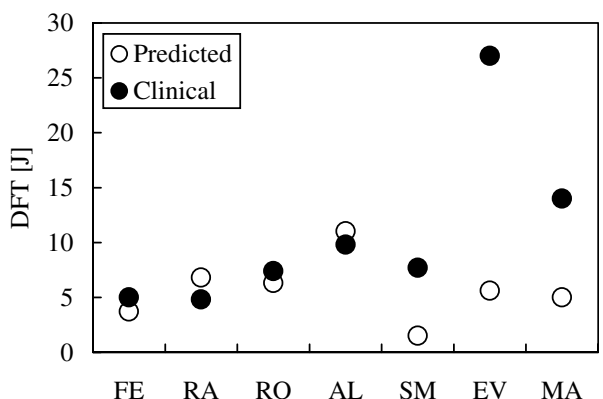

a)

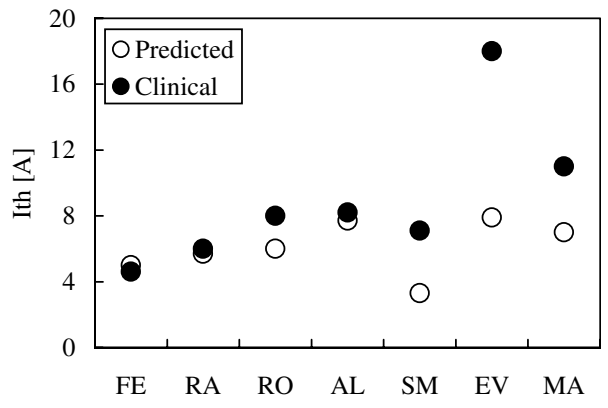

c)

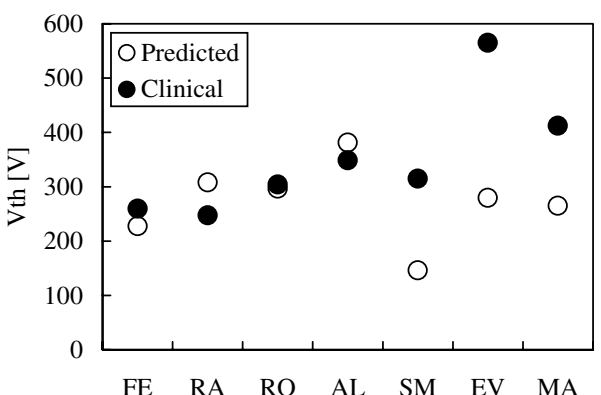

b)

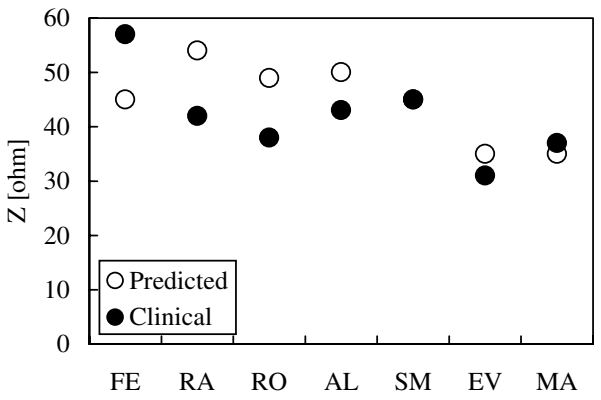

d)

Fig. 5. Model predicted and clinical defibrillation metrics: a) Energy threshold (DFT); b) Voltage threshold, $V_{t h}$; c) Current threshold, $I_{t h}$; d) Interelectrode impedance, $Z$ 
using the following identifiers: AL, FE, RA, RO, SM, MA, EV. Clinical values correspond to the lowest energy shock that defibrillated.

\section{Discussion}

This paper presents comparisons between model-predicted and clinical defibrillation metrics determined for individual patients. The model-predicted defibrillation metrics were determined from patient-specific computational models using methods similar to those developed previously to simulate electric field (current density) distributions produced by a defibrillation shock and to extract defibrillation metrics corresponding to a successful defibrillation episode [1.

Model-predicted defibrillation metrics yielded good estimates of the clinically determined metrics in four of the seven patients examined (FE, RA, RO, AL). It is significant to note that the respective clinical defibrillation metrics DFT, $V_{t h}, I_{t h}$ for these patients spanned an approximately 2-fold range, suggesting that the goodness of the achieved match was not due to the similarity of the patients examined. The model-predicted impedances all correlate well with the clinical measurements (Fig. 5d). The prediction is closest for the three patients that were not well matched by the respective threshold predictions of the model.

Each of the three patients whose clinical metrics were not well matched by the model-predicted values exhibited clinical anomalies. In one case (EV), the patient's left ventricle contained a large infarct region and exhibited a substantially compromised ejection fraction. The poor correspondence between the predicted and clinical metrics for this patient might well be due to the fact that the critical mass criterion used to extract the predicted defibrillation metrics needs to be modified to account for the substantial volume of the infarct region. In another (MA), the patient had substantial pleural effusion, which would impact the effective conductivity of the lungs and pleural space.

Although the preliminary results show that the simulated defibrillation metrics obtained using the critical mass criterion for defibrillation are generally in good agreement with clinical metrics for four of the seven patients examined, there are a number of limitations of this study. The modeling approach used can only capture geometry-based differences in defibrillation thresholds reflected in the patient population. Factors related to the underlying cellular electrophysiology enter the model only through the ad-hoc inexcitability threshold $E_{t h}$ and critical mass criterion. Hence, the model will not be able to predict defibrillation parameters for patients with cellular electrophysiology that differs substantially from the norm.

Numerical methods can also introduce errors. One source of error in the finite volume computational approach is associated with the approximation of the continuum domain with discrete, brick shaped elements. Finer meshes not only render the surfaces more smoothly, but provide more numerically accurate results. Another source of computational error is due to the values used for tissue conductivities, which may not adequately characterize the true electrical conductivity values found in the patient population. 
The correspondence between the predicted and measured defibrillation metrics observed in four of the seven patients is encouraging and provides preliminary support of the potential utility of the modeling approach. This approach may allow for patient specific presurgical planning, and may also provide a convenient computational testbed for evaluating new electrode configurations. Although these results are promising, additional subjects are needed to further validate the modeling method.

Acknowledgment. This study was supported in part by a grant from Cardiac Pacemakers, Inc., St. Paul, MN, and the Trustees of Boston University. The authors would like to thank to Dr. Michael Benser from Cardiac Rhythm Management Laboratory, Guidant, for his valuable suggestions. The excellent support provided by the Scientific Computing and Visualization Group, Boston University, is also acknowledged.

\section{References}

1. Kinst, T.F., Sweeney, M.O., Lehr, J.L., et al.: Simulated Internal Defibrillation in Humans Using an Anatomically Realistic Three-Dimensional Finite Element Model of the Thorax. J. Cardiovasc. Electrophysiol., vol.8, 537-547, 1997.

2. Min, X., Mehra, R.: Finite Element Analysis of Defibrillation fields in a Human Torso Model for Ventricular Defibrillation. Prog. Biophys. \& Mol. Biol., vol. 69, 353-386, 1998.

3. De Jongh, A.L., Entcheva, E.G., Replogle, J.A., et al.: Defibrillation Efficacy of Different Electrode Placements in a Human Thorax Model. Pace, vol. 22, 152-157, 1999.

4. Kettenbach, J., Schreyer, A.G., Okuda, et al.: 3-D Modeling of the Chest in Patients with Implanted Cardiac Defibrillator for Further Bioelectrical Simulation. In: Vannier, M.W., Inumra, K. (eds): Proc. C.A.R., Elsevier Science, 194-198, 1998.

5. Geddes, L.A., Baker, L.E.: The Specific Resistance of Biological Material: A Compendium of Data for the Biomedical Engineer and Physiologist. Med. Biol. Eng. Comput., 5:271-293, 1967.

6. Tacker, W.A. Jr., Mercer, J., Foley, P., et al.: Resistivity of Skeletal Muscle, Skin, and Lung to Defibrillation Shocks. Proc. AAMI 19th Annual Meeting, p. 81, 1984.

7. Zhou, X., Daubert, J.P., Wolf, P.D., et al.: Size of the Critical Mass for Defibrillation (abstract), Circ., vol. 80:II-531, 1989.

8. Zhou, X., Wolf P.D., Rollins P.D., et al.: Potential Gradient Needed for Defibrillation with Monophasic and Biphasic Shocks (abstract), Pace, vol. 12-651, 1989. 